Syracuse University

SURFACE

Physics

College of Arts and Sciences

2005

\title{
Temperature-Dependent Open-Circuit Voltage Measurements and Light-Soaking in Hydrogenated Amorphous Silcon Solar Cells
}

Jianjun Liang

Syracuse University

Eric A. Schiff

Syracuse University

S. Guha

United Solar Ovonic Corporation

Baojie Yan

United Solar Ovonic Corporation

Jeff Yang

United Solar Ovonic Corporation

Follow this and additional works at: https://surface.syr.edu/phy

Part of the Physics Commons

\section{Recommended Citation}

"Temperature-dependent Open-circuit Voltage Measurements and Light-soaking in Hydrogenated Amorphous Silicon Solar Cells," J. Liang, E. A. Schiff, S. Guha, B. Yan, and J. Yang, in Amorphous and Nanocrystalline Silicon Science and Technology - 2005, edited by R. Collins, P.C. Taylor, M. Kondo, R. Carius, R. Biswas (Materials Research Society Symposium Proceedings Vol. 862, Pittsburgh, 2005), A21.8 


\title{
Temperature-Dependent Open-Circuit Voltage Measurements and Light-Soaking in Hydrogenated Amorphous Silcon Solar Cells
}

\author{
Jianjun Liang, ${ }^{1}$ E. A. Schiff, ${ }^{1}$ S. Guha, ${ }^{2}$ B. Yan, ${ }^{2}$ and J. Yang ${ }^{2}$
}

${ }^{1}$ Department of Physics, Syracuse University, Syracuse, NY 13244-1130 USA

${ }^{2}$ United Solar Ovonic Corp., Troy, MI 48084 USA

\section{ABSTRACT}

We present temperature-dependent measurements of the open-circuit voltage $V_{O C}(T)$ in hydrogenated amorphous silicon nip solar cells prepared at United Solar. At room-temperature and above, $V_{O C}$ measured using near-solar illumination intensity differs by as much as $0.04 \mathrm{~V}$ for the as-deposited and light-soaked states; the values of $V_{O C}$ for the two states converge below 250 $\mathrm{K}$. Models for $V_{O C}$ based entirely on recombination through deep levels (dangling bonds) do not account for the convergence effect. The convergence is present in a model that assumes the recombination traffic in the as-deposited state involves only bandtails, but which splits the recombination traffic fairly evenly between bandtails and defects for the light-soaked state at room-temperature. Recombination mechanisms are important in understanding light-soaking, and the present results are inconsistent with at least one well-known model for defect generation.

\section{INTRODUCTION}

The open-circuit voltage $V_{O C}$ is often the simplest solar cell parameter to understand. Experimentally, $V_{O C}$ is relatively independent of the thickness of a-Si:H nip solar cells. With ideal $p$ and $n$ layers, $V_{O C}$ may be identified with bulk photocarrier recombination processes in the intrinsic material.

A simple understanding of recombination processes in a-Si:H solar cells would be valuable for two reasons. First, it would help establish which materials parameters actually determine the efficiency of working cells. Second, the metastable degradation of a-Si:H cells under illumination (the Staebler-Wronski effect) is undoubtedly mediated by photocarrier recombination - so correctly identifying the recombination processes occurring under solar illumination would be crucial to correctly identifying the microscopic mechanism underlying metastability.

In the present work, we have studied the temperature-dependence of $V_{O C}$ under strong illumination in cells deposited at United Solar Ovonic Corp.. The results exhibit an interesting "convergence" effect: the differences in $V_{O C}$ for the as-deposited and the light-soaked states at higher measurement temperatures essentially disappear below $250 \mathrm{~K}$. We show that a reasonably simple "bandtail+defect" recombination model accounts for these $V_{O C}$ measurements. In particular, the as-deposited state seems well-described by valence bandtail recombination (and neglecting defect recombination). The light-soaked state apparently involves a nearly equal combination of bandtail and defect recombination.

We do not believe that this picture is a satisfactory model for photocarrier recombination at very low excitation densities; more than two decades of research at low excitation densities has revealed daunting complexities that are certainly not accommodated by this picture. Our view is that strong illumination strongly simplifies recombination. Of course it is recombination under strong illumination that is important in metastability.

We suspect that this near-equality of bandtail and defect recombination traffic in our light-soaked a-Si:H material is a consequence of "self-limitation" to metastability: as 
recombination traffic switches from bandtails to defects, the process which generates defects shuts down. This viewpoint seems incompatible with the explanation proposed by Stutzmann, et al. [1] for the observed kinetics of the defect density $N_{d}(t)$ under illumination $N_{d}^{3}(t)=N_{d}^{3}(0)+3 C_{S W} G^{2} t ; G$ is the photogeneration rate. Their explanation assumed that defect recombination traffic predominated throughout light-soaking. We discuss alternative models for metastability kinetics elsewhere in these proceedings.

\section{TEMPERATURE-DEPENDENT $V_{O C}$ MEASUREMENTS}

For these experiments, six depositions of nip solar cells on stainless steel substrates were done at United Solar Ovonic Corp.. The $n$ and $p$ layers were the same in all depositions; the deposition time for the intrinsic layer was chosen to give intrinsic layer thicknesses from $185 \mathrm{~nm}$ to $893 \mathrm{~nm}$. The cells were not optimized for solar conversion efficiency, but the individual layers are comparable to those used in high-efficiency cells. Details of the deposition procedures have been given elsewhere [2]. As-deposited properties of the cells were measured under a solar simulator. Further studies were done using a $30 \mathrm{~mW}, 685 \mathrm{~nm}$ wavelength near-infrared laser. We chose to use this laser because its wavelength is absorbed fairly uniformly throughout the intrinsic layer of the cells, which substantially simplifies modeling of the measurements. We were able to achieve photocurrent densities in the cells that were comparable to solar illumination.

The samples were mounted in a thermoelectric cryostat. For modeling of the temperature-

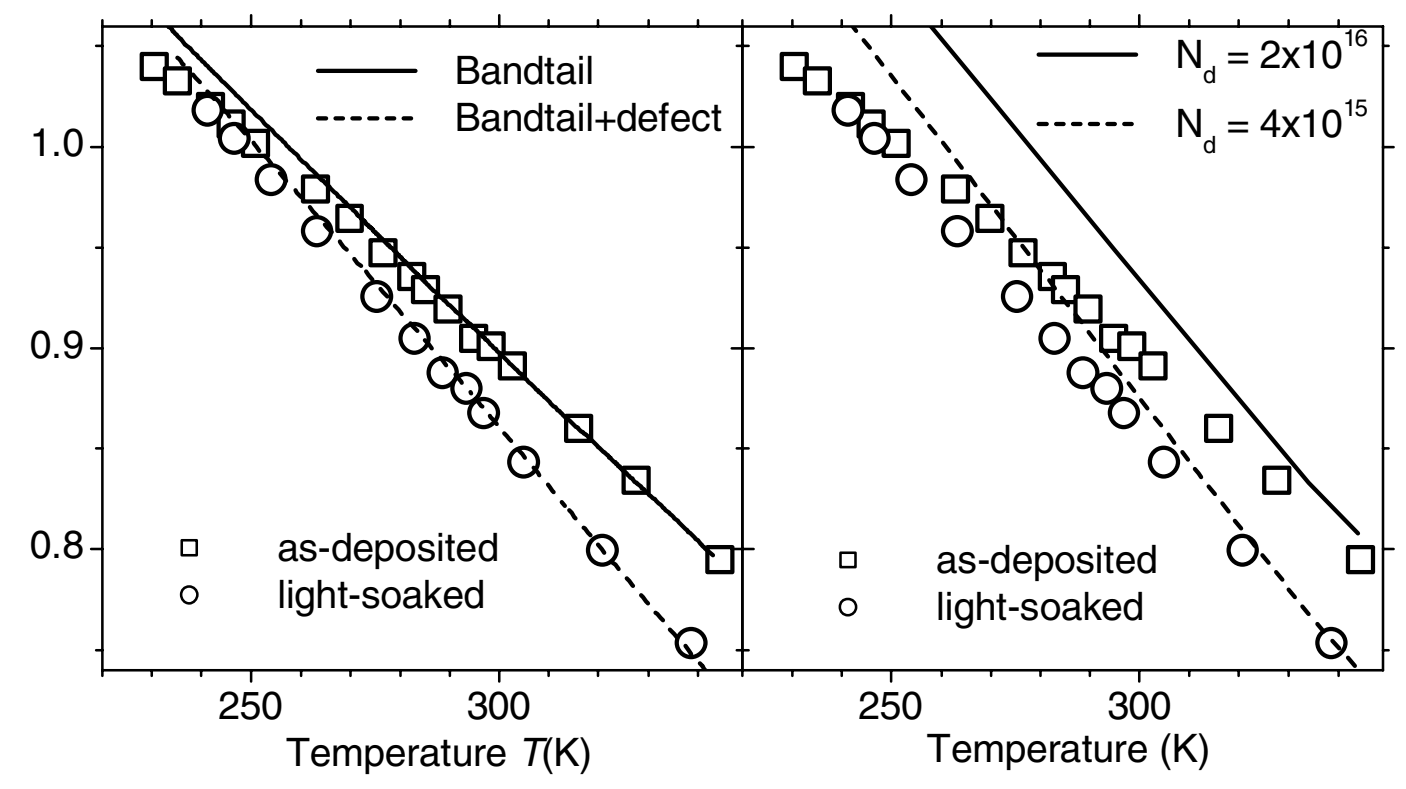

Figure 1. Temperature-dependence of the open-circuit voltage $V_{O C}$ for an a-Si:H nip solar cell (893 nm thick intrinsic layer, $295 \mathrm{~K}$ photogeneration rate $3 \times 10^{20} \mathrm{~cm}^{-3} \mathrm{~s}^{-1}$ ). The symbols represent measurements on a United-Solar cell ( $\lambda=685 \mathrm{~nm}$ photoexcitation). (left panel) The two lines represent computer calculations described in the text. The bandtail calculation only incorporates valence bandtails; the bandtail+defect calculation d incorporates the same bandtail and also a density $N_{d}=5 \times 10^{15} \mathrm{~cm}^{-3}$ of deep-levels. Note that the convergence of the measurements for lower $T$ is is fairly well represented by the modeling. (right panel) The two lines are calculated using the analytical Shockley-Read model described in the text with two defect densities. 
dependence measurements, it is essential to know the temperature-dependent bandgap $d E_{g} / d T$ of the intrinsic layers. We measured the temperature-dependent peak of the electroabsorption spectrum in one of these cells [3]. Equating $d E_{g} / d T$ with this shift, we obtained $d E_{g} / d T$ $=-4.7 \times 10^{-4} \mathrm{eV} / \mathrm{K}$, which is comparable to previous estimates based on interband optical absorption [4].

The symbols in figure 1 represent the temperature-dependence measurements of the opencircuit voltage under laser illumination for one cell; both panels show the same measurements. The several lines represent models that will be discussed subsequently. The measurements were done on a cell with $893 \mathrm{~nm}$ intrinsic layer thickness. In the as-deposited state, the photocurrent density $J$ measured at $-2 \mathrm{~V}$ and $295 \mathrm{~K}$ was $4.7 \mathrm{~mA} / \mathrm{cm}^{2}$; parameters measured under a solar illuminator were: $V_{O C}=0.982 \mathrm{~V}, J_{S C}=14.4 \mathrm{~mA} / \mathrm{cm}^{2}$, and $P=7.9 \mathrm{~mW} / \mathrm{cm}^{2}$. The light-soaked state corresponds to 176 hours of illumination at open-circuit condition at the same illumination intensity as the measurements. The sample's exposure to the laser at each temperature was 9 seconds, so the total exposure time during the temperature-dependence measurement was about 3 minutes.

Essentially the same results for $V_{O C}$ were obtained for several cells spanning our thickness range; $V_{\mathrm{OC}}$ 's very weak thickness dependence is a well-known aspect of a-Si:H solar cells [5]. Comparable temperature-dependence measurements have been published previously for the asdeposited state [6,7]. A modest decrease in $V_{O C}$ due to light-soaking is fairly commonly, if not universally, observed in a-Si:H solar cells $[8,9]$. The convergence of the $V_{O C}$ measurements for the as-deposited and light-soaked states at lower temperatures has not, to our knowledge, been noted before; it appears to complement very well the convergence at high intensities reported at $295 \mathrm{~K}$ by Pearce, et al. [8].

\section{ANALYTICAL MODELS FOR $V_{O C}$ IN nip SOLAR CELLS}

Figure 2 presents the temperaturedependence for $V_{O C}$ for three analytical models using parameters summarized in Table I and a uniform photogeneration rate $G$. A linear dependence of the bandgap $E_{g}(T)=E_{g}^{0}+\left(d E_{g} / d T\right) T$ upon temperature $T$ was also included. The $p$ and $n$ layers are assumed to be ideal. These analytical models have been confirmed using corresponding numerical simulations.

The "no traps" model simply assumes bimolecular recombination of free holes and electrons [18]; even this simplest model predicts a strong decline of $V_{O C}$ with $T$, as does the "Shockley-Read" model incorporating a single, donor-like defect level (see the appendix). Note the focus of the linear behavior at $T=0 \mathrm{~K}$; the focus voltage of about $1.9 \mathrm{~V}$ is $E_{g}^{0} / e$. The

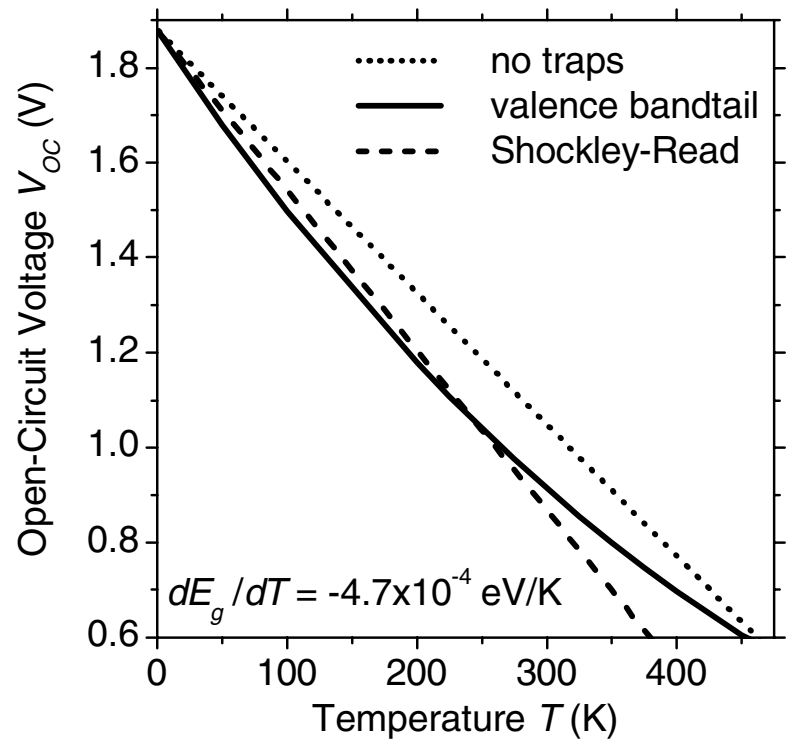

Figure 2. Temperature-dependence of the opencircuit voltage $V_{O C}$ for three models no traps, valence bandtail, and Shockley-Read; the model details and parameters are given in the text.. The photogeneration rate $G$ is $3 \times 10^{20} \mathrm{~cm}^{-3} \mathrm{~s}^{-1}$. 
"valence bandtail" model assumes an exponential valence bandtail, but no midgap defects [18]; this model predicts a parabolic behavior with temperature

\section{MODELING PARAMETERS}

Table I presents the modeling parameters used in the solar cell modeling in this paper. The bandedge and bandtail parameters have been discussed elsewhere [10]. The defect parameters are taken from Street's early work [11]. Street measured deep-trapping mobility-lifetime products $\mu \tau_{t}$ using photocarrier time-of-flight, and deep-level densities $N_{d}$ using infrared absorption and electron spin-resonance. To the best of our knowledge, later measurements (including those by some of us) do not represent a significant improvement on his estimates of the product $\left(\mu \tau_{t}\right) N_{d}$ for these photocarrier capture processes.

We have used only the parameters for the $(0 /+)$ level of the defect, and we have neglected the $D^{-}$state (and the (0/-) level) of this defect. This neglect may seem surprising, since it has been established from transient photocurrent measurements [11,12] that electrons are rapidly trapped onto neutral states $D^{0}$ to form negatively charged $D^{-}$states. Many experiments have explored the density-of-states associated with this (0/-) level. Essentially all of these measurements have been done at fairly low photoexcitation levels; among other reasons, experimenters have been interested in reducing light-soaking during measurements. For measurements done under strong (nominally solar) illumination, electron deep-trapping becomes undetectable in a-Si:H $[13,14]$. There is no well-accepted explanation for this quenching of the deep-trapping process by illumination.

We have neglected the temperature-dependence of all of these parameters except $E_{g}$. Because $V_{O C}$ depends logarithmically on most of these parameters, this neglect isn't likely to lead to significant error for the present work. Quite different parameters have been used by other modelers $[15,16]$; we cannot give a fuller discussion of the different choices here.

\section{DISCUSSION}

The lefthand panel of figure 1 presents a full computer calculation of $V_{O C}(T)$ using the AMPS-1D computer program [17] and the parameters from Table I. The $p$ and $n$ layer parameters for the computer calculations were "ideal," and varying them substantially had no noticeable effect on $V_{O C}$. The values for $E_{g}, N_{C}$, and $N_{V}$ were obtained from fittings to our earlier $V_{O C}(T)$ measurements [10], so the success in fitting to the as-deposited measurements is unsurprising; these parameters also accounted well for thickness-dependent power measurements which were not fitted, and this is the main justification for their use.

Table I: Modeling Parameters [10,11]

\begin{tabular}{|l|l|l|}
\hline$b_{d n}$ & $4.0 \times 10^{-8} \mathrm{~cm}^{3} \mathrm{~s}^{-1}$ & $\mathrm{e} \rightarrow \mathrm{D}^{+}$. Calculated from $\left(\mu \tau_{t}\right) N_{d}$ assuming $\mu_{n}=2 \mathrm{~cm}^{2} / \mathrm{Vs}$. \\
\hline$b_{d p}$ & $7.5 \times 10^{-9} \mathrm{~cm}^{3} \mathrm{~s}^{-1}$ & $\mathrm{~h}^{+} \rightarrow \mathrm{D}^{0}$. Calculated from $\left(\mu \tau_{t}\right) N_{d}$ assuming $\mu_{p}=0.3 \mathrm{~cm}^{2} / \mathrm{Vs}$. \\
\hline$E_{g}$ & $1.74 \mathrm{eV}$ & Electrical bandgap (from $\left.V_{O C}(T)\right)$. \\
\hline$d E_{g} / d T$ & $-4.7 \times 10^{-4} \mathrm{eV} / \mathrm{K}$ & From electroabsorption; see text. \\
\hline$N_{v}$ & $4 \times 10^{20} \mathrm{~cm}^{-3}$ & Valence band effective-density-of-states (from $V_{O C}(T)$ ). \\
\hline$N_{c}$ & $4 \times 10^{20} \mathrm{~cm}^{-3}$ & Arbitrarily set equal to $N_{v}$. \\
\hline$\Delta E_{v}$ & $40 \mathrm{meV}$ & Width of exponential valence bandtail; from hole drift-mobility. \\
\hline$b_{t p}$ & $1.3 \times 10^{-9} \mathrm{~cm}^{3} \mathrm{~s}^{-1}$ & $\mathrm{~h}^{+} \rightarrow \mathrm{T}^{0}$ (valence bandtail). \\
\hline$b_{t n}$ & $1.0 \times 10^{-9} \mathrm{~cm}^{3} \mathrm{~s}^{-1}$ & $\mathrm{e}^{-} \rightarrow \mathrm{T}^{+}$(valence bandtail). \\
\hline
\end{tabular}


For these calculations we included the temperature-dependent absorption coefficient of the samples as well as the temperature-dependent bandgap; we inferred the absorption coefficient at $685 \mathrm{~nm}$ from photocurrent density measurements on samples with five different thicknesses at $-2 \mathrm{~V}$ bias. The solid line in the figure represents the model incorporating the valence bandtail, but neglecting defects altogether. The dashed line represents the model incorporating $N_{d}=$ $5 \times 10^{15} \mathrm{~cm}^{-3}$ defects as well as the bandtail. We placed this level $1.1 \mathrm{eV}$ below the conduction bandedge, but the exact level position had little effect on the calculation. A similar "bandtail+defect" computer model was studied previously to explore the correlation of the fillfactor and $V_{O C}$ as light-soaking proceeds [9].

As is evident, the fairly rapid "convergence" of the measurements at lower temperatures for as-deposited and light-soaked a-Si:H is fairly well described by these models. At the lowest temperatures, the experimental results are still lower than the model predictions at lower temperatures. We believe that this effect is due to limitation of $V_{O C}$ by the $p$-layer. A very clear saturation of $V_{O C}$ for temperatures below $200 \mathrm{~K}$ was reported previously, and was interpreted as due to $p$-layer limitation [7]. We did not attempt to fit our data by modifying the $p$-layer parameters.

The right panel of figure 1 illustrates the temperature-dependence of $V_{O C}$ predicted by the analytical Shockley-Read ("defects only") model. Since $G(T)$ does vary with depth in the actual sample, we used its value at the center of the intrinsic layer for the calculations. The two defect densities used for the calculations in the figure were chosen to give good agreement with the experiment at higher temperatures. As can be seen, the Shockley-Read model gives a poor account for the temperature-dependence of $V_{O C}$. We believe that the measurements largely exclude the Shockley-Read picture as an alternative to the bandtail+defect model.

The authors thank Steven Hegedus for discussions and access to unpublished measurements. This work has been supported through the Thin Film Photovoltaics Partnership of the National Renewable Energy Laboratory (NDJ-2-30630-24 and ZDJ-2-30630-19).

\section{APPENDIX: A SHOCKLEY-READ MODEL}

One of the simplest models for $V_{O C}$ of an nip solar cell assumes that electrons and holes recombine through a density $N_{d}$ of donor-type deep levels $(0 /+)$; the defects are assumed to be neutral in the dark. We first calculate the densities $n$ and $p$ of mobile electrons and holes. For uniform photogeneration rate $G$, the rate equations are:

$$
\frac{d n}{d t}=G-b_{d n} n P,(1) \quad \frac{d p}{d t}=G-b_{d p} p\left(N_{d}-P\right),
$$

where $P$ is the density of holes occupying the defects under illumination, and $b_{d n}$ and $b_{d p}$ describe the capture of electrons and holes by positively charged and neutral defects, respectively. We are neglecting the possibility of re-emission of carriers that have been captured; the defect thus acts as a recombination center, and not as a "trap." Charge neutrality requires $p+P=n$. If we assume $p<<P<<N_{d}$ (valid for $G<<b_{d n} N_{d}^{2}$ ), we obtain for the steady-state solutions:

$$
\begin{gathered}
b_{d p} p N_{d}=b_{d n} n P=G, \\
n=P=\sqrt{G / b_{d n}} \text {, and } p=G /\left(N_{d} b_{d n}\right) .
\end{gathered}
$$

These solutions can be rewritten in terms of the electron and hole quasi-Fermi levels, which are defined $E_{F n} \equiv E_{c}+k_{B} T \ln \left(n / N_{c}\right)$ and $E_{F p} \equiv E_{v}-k_{B} T \ln \left(p / N_{v}\right)$, where $N_{c}$ and $N_{v}$ are the 
effective densities of states at the conduction bandedge $E_{c}$ and valence bandedge $E_{v}$ :

$$
E_{F n}=E_{c}-\left(k_{B} T / 2\right) \ln \left(b_{d n} N_{c}^{2} / G\right) \text {, (4) } \quad E_{F p}=E_{v}+\left(k_{B} T\right) \ln \left(b_{d p} N_{v} N_{d} / G\right) .
$$

For nip solar cells with "ideal" $p$ and $n$ layers, the open-circuit voltage may be approximated by $e V_{o c}=E_{F n}-E_{F p}$; the relationship has been validated by numerical studies in several cases [18]. We obtain:

$$
e V_{o c}=E_{g}-\left(k_{B} T / 2\right) \ln \left(b_{d n} N_{c}^{2} / G\right)+k_{B} T \ln \left(b_{d p} N_{v} N_{d} / G\right)
$$

It is worth noting that the position of the (0/+) level in the gap does not appear in these expressions, which reflects our neglect of electron and hole emission from deep levels.

\section{REFERENCES}

1. M. Stutzmann, W. B. Jackson, and C. C. Tsai, Phys. Rev. B 32, 23 (1985).

2. J. Yang, A. Banerjee, and S. Guha, Appl. Phys. Lett. 70, 2975 (1997).

3. L. Jiang, Q. Wang, E. A. Schiff, S. Guha, and J. Yang, Appl. Phys. Lett. 72, 1060 (1998).

4. G. D. Cody, T. Tiedje, B. Abeles, B. Brooks, and Y. Goldstein, Phys. Rev. Lett. 47, 1480 (1982).

5. K. Mitchell, D. Tanner, S. Vasquez, D. Willett and S. Lewis, in Proc. 18th Photovoltaic Specialists Conference (IEEE, 1985), 914.

6. S. S. Hegedus and W. N. Shafarman, Prog. Photovolt: Res. Appl. 12, 155 (2004)

7. Y. Lee, A. Ferlauto, and C.R.Wronski,in Proc. 26th Photovoltaic Specialists Conference (IEEE, 1997), 683.

8. J. Pearce, R. Koval, A. Ferlauto, R. Collins, C. Wronski, J. Yang, and S. Guha, Appl Phys. Lett. 77, 19 (2000).

9. B. Yan, J. Yang, and S. Guha, Appl. Phys. Lett. 83, 782 (2003).

10. K. Zhu, J. Yang, W. Wang, E. A. Schiff, J. Liang, and S. Guha, in Amorphous and Nanocrystalline Silicon Based Films - 2003, edited by J. R. Abelson, et al. (Materials Research Society, Symp.Proc. Vol. 762, Pittsburgh, 2003), 297.

11. R. A. Street, J. Zesch, and M. J. Thompson, Appl. Phys. Lett. 43, 672 (1983).

12. H. Antoniadis and E. A. Schiff, Phys. Rev. B 44, 3627 (1991)..

13. D. Ritter, E. Zeldov, and K. Weiser, Phys. Rev. B. 38, 8297 (1988).

14. D. Han, E. A. Schiff, and M. Silver, Phys. Rev. B. 48, 8658 (1993).

15. U. Dutta and P. Chatterjee, J. Appl. Phys. 96, 2261 (2004).

16. R. Schropp and M. Zeman, Amorphous and Microcrystalline Silicon Solar Cells: Modeling, Materials, and Device Technology (Kluwer, Boston, 1998).

17. H. Zhu and S. J. Fonash, collected in Amorphous and Microcrystalline Silicon Technology 1998, edited by R. Schropp, et al. (Materials Research Society, Symp. Proc. Vol. 507, Pittsburgh, 1998), 395.

18. E. A. Schiff, Solar Cells and Solar Energy Materials 78, 567 (2003). 